\title{
Immune system under assault in Massachusetts
}

\author{
Scott W. Umlauf and Una S. Ryan
}

A war is being fought for therapeutic control of the immune system. The old empire of global immunosuppression with the dual defects of toxicity and lack of specificity are giving way to a new confederation of precisely targeted therapies, as we enter the postcyclosporine(Cs) era. This was made evident in four talks (Fig. 1) presented at the immune suppression section of the Massachusetts Biotechnology Council Symposium (May 8, 1996, Cambridge, MA).

Bill Paul's (Office of AIDS research, NIH, Bethesda, MD) talk led the opening session with an elegant example of how the actions of even a single cytokine can be separated into unique components, allowing the possibility of highly specific therapies. In dissecting the mechanisms of signaling via the IL-4 receptor (IL-4R), using the now familiar arsenal of molecular biology tricks, Paul and his coworkers demonstrated that the two fundamental outcomes of IL- 4 binding to its receptor-division or differentiation-arise from biochemically distinct domains on the protein. The motif that causes proliferation is shared with other growth factor receptors, such as the insulin receptor, whereas differentiation signals are sent, at least in large part, by the JAK-STAT pathway of kinase/transcription factor pairing. Thus, there is the potential for drugs that modulate the differentiation pathway (for example, switching to IgE production to block allergy induction) while leaving the proliferative arm intact.

Lee Nadler (Dana-Farber Institute, Harvard Medical School, Boston, MA) focused his talk on the attempt to solve the difficult therapeutic problem of graft-versus-host (GVH) disease in bone-marrow transplantation. This dilemma is caused by the presence of $\mathrm{T}$ cells and natural killer cells in the donor marrow that can attack the host tissue. This disease is particularly frustrating for clinicians because of the need for highly matched marrow, and the inability of current immunosuppressive therapies, such as cyclosporin A (CsA), to combat it. The approach of Nadler and coworkers relies on the extensive literature regarding the induction of long-term antigenic tolerance by $\mathrm{T}$ cell anergy (dormancy of an immune response). Because it has long been known that costimulatory ligands on antigen-pre-

Scott W. Umlauf is a senior scientist and Una S. Ryan is president and COO of T Cell Sciences, 115 Fourth Avenue, Needham, MA 021942725 (73400.1566@compuserve.com).

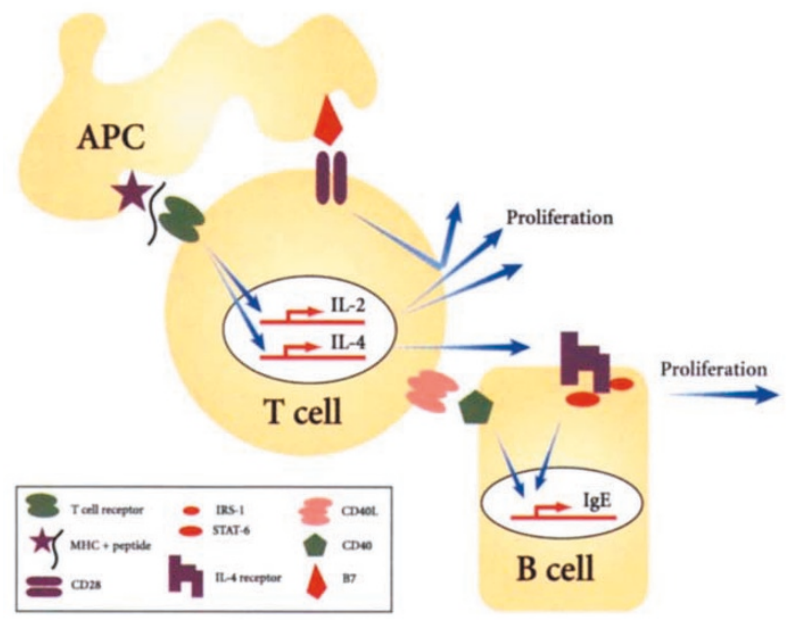

Figure 1. Four approaches to immunoregulation: $T$ cells are signaled through the T-cell receptor (TCR) by antigen-MHC complexes on the surface of antigen-presenting cells (APC). This leads to the transcription of cytokines, such as IL-2 and IL-4. If the CD28 molecule on T cells is costimulated by B7 on the APC, IL-2 production is augmented, and the cells survive and proliferate. IL-4 signaling through its receptor affects both proliferation and differentiation (e.g., synthesis of $\mathrm{IgE}$. These effects are mediated through independent domains of the IL-4 receptor. TCR signaling aiso induces CD40L on the surface of T cells. CD40L interaction with CD40 is critical for the survival and differentiation of B cells.

senting cells (APCs) are critical for the full activation of $T$ cells, Nadler's team have devised anergy-inducing presenters by transfecting immortalized, B7-negative cells with an HLA from a recipient. The marrow was then treated with these cells in order to induce anergy in potentially alloreactive cells. Key in vitro results for the procedure have been promising: After several days in culture, anergized marrow cells have been unable to be restimulated with the tolerizing HLA, even when presented on costimulation-competent APCs. However, the anergized cells can respond to a third party HLA. Unfortunately, however, the two patients who received anergized marrow did not survive long enough to provide meaningful clinical endpoints.

David Thomas (Biogen, Cambridge, MA) presented a thorough description of the potential utility of an antibody that blocks the action of the CD40 ligand (CD40L). The clinical significance of this molecule to the immune system has been clearly demonstrated: A human disease, the hyper-IgM syndrome, has been mapped to mutations in the gene. Males afflicted with this X-linked malady are incapable of class-switching antibodies to anything but IgM, and are severely immunocompromized as a result.

Furthermore, CD40L has also been shown to be critical for the survival of B cells when receiving antgenic stimulation, both initially and during affinity maturation in the germinal centers. Thomas presented a summary of the extensive number of rodent disease models that have been successfully treated with an anti-CD40 antibody, and the results of primate studies that reveal an unusually long serum half-life. Interestingly, this anti-CD40 monoclonal antibody may be one of the few that does not need to be humanized for therapeutic utility, because it blocks the antiisotype response. Although effcacy in blocking antibody responses is expected, a delicate balance may have to be maintained so that memory $B$ cells to common pathogens are not lost.

Finally, we (T Cell Sciences, Needham, MA) presented progress in finding novel inhibitors of IL-2 transcription. Using a cell-based screen, a group of small-molecule immunosuppressives has been identified from an organic array library synthesized at ArQule (Medford, MA). Using a focused array of biochemical secondary assays, we have been able to home in on the site of action of the most potent lead compounds. Importantly, these leads do not act on calcineurin, the widely expressed phosphatase that is the site of action of CsA and FK-506. At present, the interaction between the Ras and JNK pathways appears to be the site of action of these compounds. Although these pathways are found in many cell types, lymphocyte-specific components apparently do exist-as seen, for example, in the calcium-dependent activation of JNK. Concurrent with the determination of biochemical mechanism, animal studies have demonstrated inhibition of a delayed-type hypersensitivity response to a peptide antigen with one of the lead compounds.

Taken together, these four approaches, as well as several others not covered at this symposium, demonstrate the new principle for the central goal of therapeutic immunoregulation: for each immunopathogenic state, an optimum target and therapy. Or, from the many, one. 